\title{
Molecularly-imprinted silica nanoparticles for rapid and selective detection of atenolol in artificial urine samples
}

\author{
Adem Zengin \\ Van Yuzuncu Yil University, Faculty of Science, Department of Chemistry, 65080 Van-Turkey, \\ ademzengin@yyu.edu.tr, ORCID: 0000-0002-6889-5387
}

\begin{abstract}
A B S T R A C T
Herein, a novel molecularly imprinted polymer was synthesized on silica nanoparticles via surface imprinting approach for rapid, sensitive and selective detection of atenolol in artificial urine samples. For this purpose, silica nanoparticles were firstly modified with methacryloxy group for surface initiated polymerization and then, polymerization was carried out in the presence of 2-hydroxyethyl methacrylate (functional monomer), ethylene glycol dimethacrylate (cross-linker), azobisisobutyronitrile (initiator), atenolol (template) and acetonitrile (porogen). The surface characterization of imprinted nanoparticles indicated that a thin polymer layer was grafted on the silica nanoparticles. The rebinding properties of the imprinted nanoparticles were investigated in detail and the results revealed that the imprinted nanoparticles had high adsorption capacity (32.06 mg/g), fast adsorption kinetics (15 min for equilibration), high imprinting factor (4.14) towards atenolol and good regeneration ability. The imprinted nanoparticles were also used as selective sorbent for selective extraction and determination of atenolol in artificial urine samples. The results showed that the proposed method good recovery percentages (98.6 \%-100.1\%) with low standard deviations (less than 4.4\%). It is believed that the atenolol-imprinted silica nanoparticles can be used as an alternative sorbent for selective quantification of atenolol in artificial urine samples.
\end{abstract}

\section{ARTICLE INFO}

\section{Research article}

Received: 12.01 .2021

Accepted: 11.03.2021

Keywords:

molecularly-imprinted

polymers,

surface imprinting,

silica nanoparticles,

atenolol,

artificial urine

\section{Introduction}

Molecular imprinting is a powerful method for the creation of selective binding sites for target molecules in polymer networks [1]. The formed binding sites are complementary to target molecules in shape, size, and functional group orientations [2]. Molecularly-imprinted polymers (MIPs) can be easily synthesized in the presence of functional monomer, template (target) molecule, cross-linker, initiator and porogen (solvent). Due to the superior features of MIPs such as high thermal, mechanical and chemical stability, long durability without loss their selective recognition ability and robustness, MIPs are generally used in separation/purification technologies [3], catalysis [4], sensors [5], environmental and food testing [6] and many other practical applications [7].

Atenolol is a type of $\beta$-blocker and generally used for treatment of cardiovascular disorders such as angina pectoris, myocardial infarction, cardiac arrhythmias, and hypertension [8]. Atenolol reduces tremors and heart attacks and often used by athletes for improving their performance. Therefore, atenolol is classified as anti-doping agent and the usage of atenolol is banned by the World Anti-Doping Agency
(WADA) [9]. As a result, determination of atenolol in biological samples is an important issue.

There have been several analytical methods such as high performance liquid chromatography (HPLC) [10], liquid chromatography-mass spectrometry (LC-MS) [11], gas chromatography-mass spectrometry (GC-MS) [12], potentiometry [13], spectrofluorimetry [14], and enzymelinked immunoassay (ELISA) [15] reported for determination of atenolol in different biological samples. The aforementioned chromatographic methods are generally suffer from pure selectivity due to the presence of potential interferences which limits sensitivity of the methods. Moreover, chromatographic methods requires high cost equipment, large solvent consumption, complicated sample pre-treatment steps, requiring highly skilled operators. ELISA is the most preferred method for determination of drugs in clinical applications but the method has some drawbacks such as cross-reactivity of endogenous molecules, single use, the need for a highly skilled operators. Especially, spectrofluorimetric methods also suffer from potential fluorescent interferences in biological samples. Therefore, an 
effective, sensitive and selective analytical method is necessary for the determination of atenolol in biological samples.

Herein, a novel analytical method was presented for selective extraction and sensitive determination of atenolol in artificial urine sample based on surface imprinting approach. For this purpose, 3- methacryloxypropyl trimethoxysilane (MPS) modified silica nanoparticles (MPS@SiO 2 NPs), 2hydroxyethly methacrylate (HEMA), atenolol, ethylene glycol dimethacrylate (EGDMA), azobisisobutyronitrile $(\mathrm{AIBN})$, and acetonitrile (ACN) were used as supporting material, template molecule, cross-linker, initiator and porogen, respectively. Surface characterization was conducted to verify grafting of the imprinted polymer. Rebinding properties of atenolol on the imprinted/nonimprinted polymer were investigated in detail. Moreover, the prepared atenolol-imprinted nanoparticles (MIP@SiO 2 NPs) were used as selective sorbent for extraction of atenolol and its quantification by spectrofluorimetry in artificial urine samples.

\section{Materials and methods}

3- methacryloxypropyl trimethoxysilane (MPS), 2hydroxyethly methacrylate (HEMA), tetraethyl orthosilicate (TEOS) and ethylene glycol dimethacrylate (EGDMA) were purchased from Sigma-Aldrich and used as received unless otherwise noted. Azobisisobutyronitrile (AIBN) was provided by Across Organics and recrystallized twice from methanol. Atenolol, propranolol and pindolol were supplied by Alfa Aesar Chemicals and used as received.

\subsection{Preparation of $\mathrm{SiO}_{2} \mathrm{NPS}$}

$\mathrm{SiO}_{2}$ NPs were synthesized via well-known Stöber method [16]. Briefly, $2.5 \mathrm{~mL}$ TEOS was added to $50 \mathrm{~mL}$ absolute ethanol and then, $600 \mu \mathrm{L} \mathrm{NH} \mathrm{NH}_{4} \mathrm{OH}$ was added to the mixture. The solution was stirred at $60{ }^{\circ} \mathrm{C}$ for overnight. The resultant milky solution was centrifuged at $6500 \mathrm{rpm}$ for $10 \mathrm{~min}$ to recovery the silica nanoparticles. The nanoparticles were repeatedly washed with ethanol. Lastly, the nanoparticles were dried in a vacuum oven.

\subsection{Preparation of MPS@SiO 2 NPs}

$100 \mathrm{mg} \mathrm{SiO}_{2}$ NPs were dispersed in $25 \mathrm{~mL}$ toluene via ultrasonication for $10 \mathrm{~min}$ at room temperature. $500 \mu \mathrm{L}$ of MPS was added to the mixture and the mixture was stirred 18 $\mathrm{h}$ at room temperature. MPS modified silica nanoparticles were collected by centrifugation at $6500 \mathrm{rpm}$ for $10 \mathrm{~min}$ and washed with plenty of toluene, toluene:methanol and methanol, respectively. Lastly, the nanoparticles were dried in a vacuum oven.

\subsection{Preparation of MIP/NIP@SiO 2 NPs}

MIP@SiO 2 NPs were prepared via surface initiated free radical polymerization. Atenolol (0.4 mmol) and HEMA (1.6 mmol) were added to $10 \mathrm{~mL}$ acetonitrile. The mixture was stirred at room temperature for $4 \mathrm{~h}$ to form pre-polymerization mixture. $8.0 \mathrm{mmol}$ EGDMA and $0.015 \mathrm{mmol}$ AIBN were added to the solution. Subsequently, 100 mg MPS@ $\mathrm{SiO}_{2}$ was dispersed in the mixture and polymerization was carried out at $60{ }^{\circ} \mathrm{C}$ for $8 \mathrm{~h}$ after degassing the mixture with nitrogen for 15 min on an ice-bath. After polymerization, the mixture was diluted by $10 \mathrm{~mL} \mathrm{ACN}$ and the mixture was centrifuged. The imprinted atenolol was removed from the nanoparticles by washing with methoanol:acetic acid (7/3, v/v) in a Soxhlet extractor. Same protocol was applied to prepare NIP@SiO particles except in the absence of atenolol.

\subsection{Rebinding experiments}

$1.5 \mathrm{mg}$ of MIP/NIP@ $\mathrm{SiO}_{2}$ NPs dispersed in acetonitrile solution of atenolol at concentration range between 0.1 $\mathrm{mg} / \mathrm{mL}-1.0 \mathrm{mg} / \mathrm{mL}$. The mixture was stirred on an orbital shaker for different time intervals (1-45 min). After rebinding, the nanoparticles were collected by centrifugation. The supernatant was filtered through cellulose acetate membrane and the remaining concentration of atenolol was determined by spectrofluorimetry ( $\lambda_{\text {excitation}}: 274 \mathrm{~nm}, \lambda_{\text {emission }}: 300 \mathrm{~nm}$ ). The adsorption capacities of nanoparticles were calculated according to the formula $Q=\left(C_{0}-C_{s}\right) . V / m$ where $C_{0}$ and $C_{s}$ are atenolol concentration before and after rebinding, respectively, $V$ is the volume of the solution, and $m$ is the mass of the nanoparticles.

\subsection{Selectivity test}

In order to test the selectivity of MIP@SiO $\mathrm{NPs}_{2}$, propranolol and pindolol were selected as structural analogues. $1.5 \mathrm{mg}$ ${\mathrm{MIP} @ \mathrm{SiO}_{2}}_{2}$ was separately dispersed in $0.6 \mathrm{mg} / \mathrm{mL}$ concentrations of atenolol, propranolol and pindolol solutions in acetonitrile. The mixtures were stirred on an orbital shaker for $15 \mathrm{~min}$ at room temperature and the concentration was determined by spectrofluorimetry.

\subsection{Determination of atenolol in spiked artificial urine samples}

Artificial urine sample was prepared according to published procedure [17] and $\mathrm{pH}$ of the artificial urine sample was adjusted to $\mathrm{pH} 5.10 \mathrm{~mL}$ of artificial urine sample was spiked with different concentration $(0.2 \mu \mathrm{g} / \mathrm{mL}-2.5 \mu \mathrm{g} / \mathrm{mL})$ of standard stock solution of atenolol in acetonitrile. Then, $15 \mathrm{mg}$ MIP@SiO 2 NPs were added to the artificial urine sample and the mixture was stirred for $15 \mathrm{~min}$ at room temperature. The nanoparticles were removed from solution by centrifugation and washed with plenty of water. Then, the nanoparticles was washed with methanol:acetic acid $(7 / 3, \mathrm{v} / \mathrm{v})$ to remove the rebound atenolol and concentration of atenolol in the supernatant was determined by spectrofluorimetry. 


\section{Instruments}

The attenuated total reflectance- Fourier transform infrared (ATR-FTIR) analysis was carried out by a Thermo Nicolet 6700 spectrometer and the spectra were collected at a resolution $4 \mathrm{~cm}-1$ after 256 scans. The surface morphology and nanoparticle diameter were determined by transmission electron microscopy (TEM, JEOL JEM 1400). A spectrofluorometer (Shimadzu RF-6000) was used for rebinding experiments.

\section{Results and discussion}

\subsection{Fabrication of MIP@SiO ${ }_{2} \mathrm{NPs}$}

The synthesis procedure of MIP@SiO 2 NPs is schematically shown in Figure 1. Firstly, $\mathrm{SiO}_{2} \mathrm{NPs}$ were prepared by Stöber method and then, modified with MPS to initiate polymerization on the silica particles. The atenolol-imprinted particles were fabricated through surface-initiated free radical polymerization in the presence of HEMA, atenolol, EGDMA, AIBN and ACN. After polymerization, the imprinted atenolol was removed from the polymer network by methanol:acetic acid mixture and the resultant polymer network on the $\mathrm{SiO}_{2}$ NPs consists specific cavities that complementary to the atenolol in terms of size, shape and functionality.

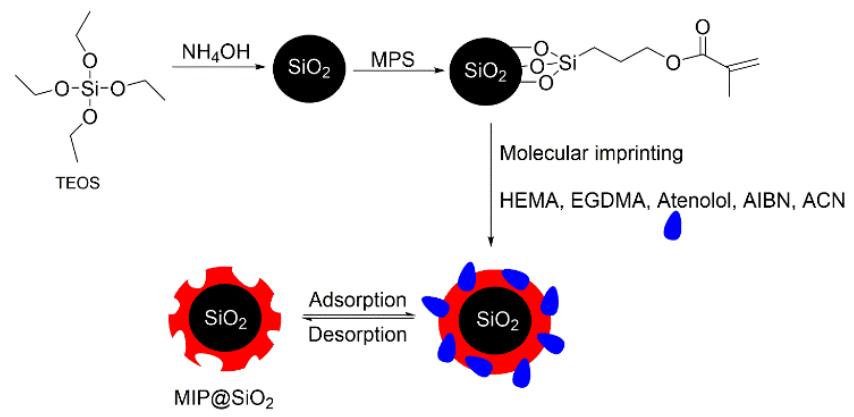

TEOS: tetraethyl orthosilicate

MPS: 3- methacryloxypropyl trimethoxysilane

HEMA: 2-hydroxyethly methacrylate

EGDMA: Ethylene glycol dimethacrylate

AIBN: Azobisisobutyronitrile

ACN: Acetonitrile

Figure 1. Schematic illustration for preparation of ${\mathrm{MIP} @ \mathrm{SiO}_{2}}_{2}$ NPs.

\subsection{Characterization of MIP@SiO 2 NPs}

The chemical characterization of silica particles before and after modification were carried out by ATR-FTIR spectroscopy. For bare silica particles (Figure 2a), the band at $1071 \mathrm{~cm}^{-1}$ could be assigned as Si-O-Si stretching vibrations of tetrahedral silica structures. After covalent attachment of MPS onto silica particles, a new peak recorded at $1709 \mathrm{~cm}^{-1}$ which could be attributed to the carbonyl group of MPS indicating the covalent binding of MPS. The ATR-FTIR spectrum of MIP@SiO ${ }_{2} \mathrm{NPs}$ (Figure 2c), the band at $1716 \mathrm{~cm}^{-}$ 1 associated with carbonyl groups of HEMA and the absorption bands at around $3426 \mathrm{~cm}^{-1}$ and $2956-2882 \mathrm{~cm}^{-1}$ could be assigned as hydroxyl groups and methyl/methylene groups of HEMA indicating the successful grafting of polymer on the silica particles $[18,19]$.

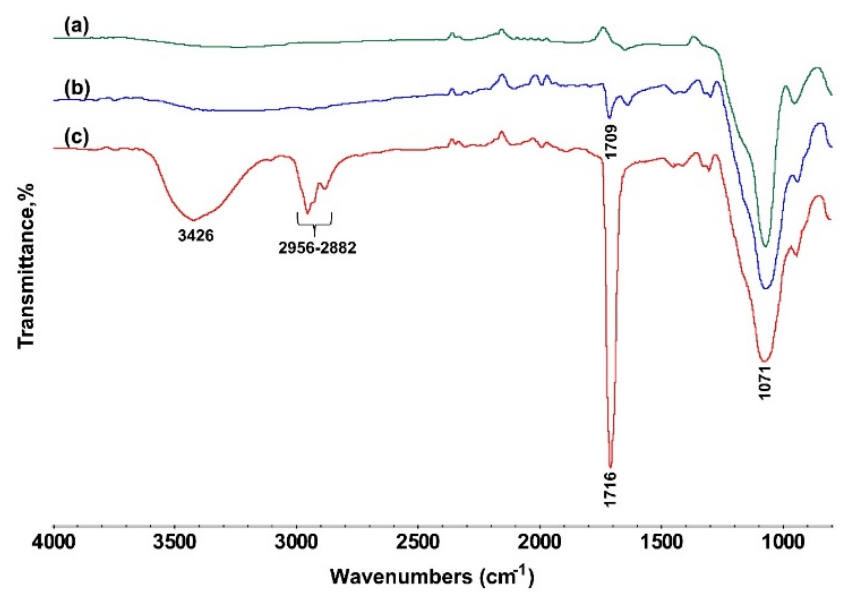

Figure 2. ATR-FTIR spectra of (a) bare $\mathrm{SiO}_{2}$, (b) MPS@ $\mathrm{SiO}_{2}$ and (c) $\mathrm{MIP} @ \mathrm{SiO}_{2} \mathrm{NPs}$.

The size and morphology of ${\mathrm{MIP} @ \mathrm{SiO}_{2}}^{\mathrm{NPs}}$ were determined by TEM. As shown in low magnification TEM image of MIP@SiO ${ }_{2}$ NPs (Figure 3a), the particles have spherical morphology and no agglomeration occurred after polymerization. Moreover, the particles consist $\mathrm{SiO}_{2}$ core with a diameter of $93 \mathrm{~nm}$ and a homogenous polymer layer with a relatively bright layer and thickness of $21 \mathrm{~nm}$ (Figure 3b).

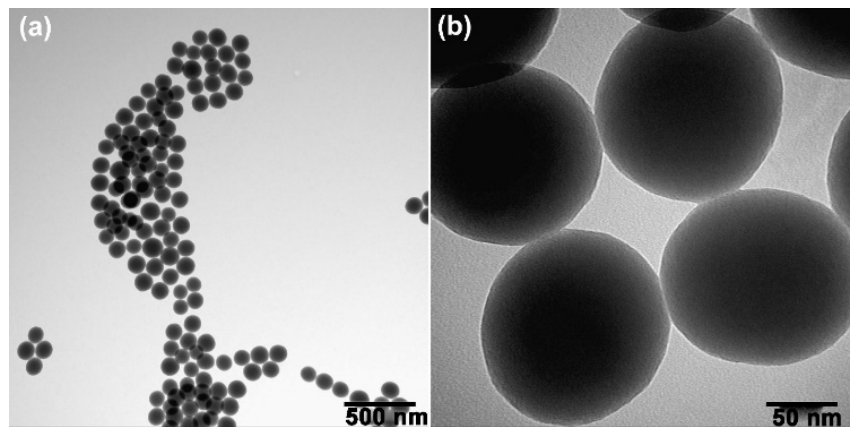

Figure 3. TEM images of $\mathrm{MIP@SiO}$ NPs with low (a) and high (b) magnifications.

\subsection{Rebinding properties of atenolol on MIP/NIP@SiO $2 \mathrm{NPs}$}

The adsorption kinetics for atenolol MIP/NIP@SiO 2 NPs are shown in Figure 4a. The adsorption capacities of both particles increased with increasing adsorption time and reached an equilibration at 15 min adsorption time. Moreover, the adsorption capacity of MIP@SiO ${ }_{2}$ NPs was higher than $\mathrm{NIP@SiO}{ }_{2} \mathrm{NPs}$ for all adsorption times implying the 
presence of specific recognition cavities on the imprinted particles. Meanwhile, the lower adsorption capacities of $\mathrm{NIP} @ \mathrm{SiO}_{2} \quad \mathrm{NPs}$ could be mainly occurred non-specific interactions between atenolol and polymer network [19].

The binding isotherms of MIP/NIP@SiO ${ }_{2}$ NPs are given in Figure 4b. It is obviously seen that the adsorption capacity of MIP/NIP@SiO ${ }_{2}$ NPs towards atenolol increased with increasing initial concentration of atenolol and reached saturation when the initial concentration was $0.6 \mathrm{mg} / \mathrm{mL}$. The adsorption capacity of MIP@SiO 2 NPs was 32.06 mg/g, 4.14 times higher than that of NIP@SiO ${ }_{2}$ NPs which is a natural result of the presence of specific binding sites on ${\mathrm{MIP} @ \mathrm{SiO}_{2}}_{2}$ NPs.
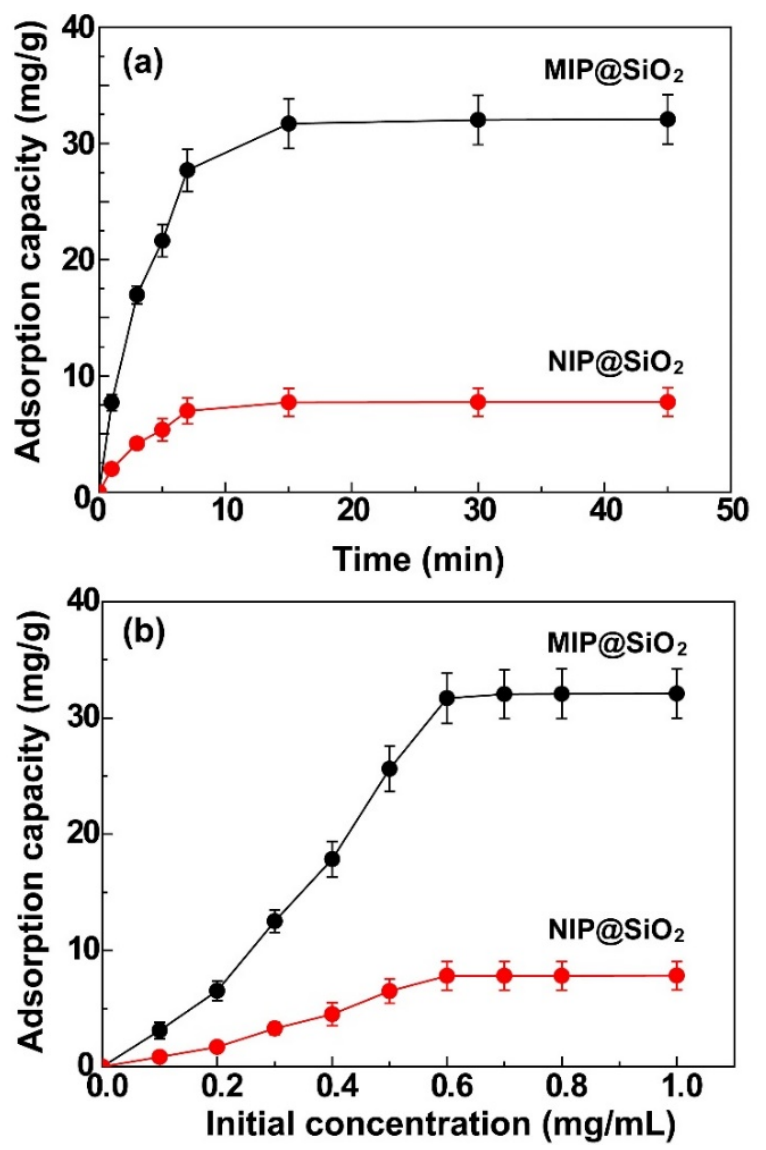

Figure 4. (a) Adsorption kinetics for MIP/NIP@SiO 2 NPs and (b) Adsorption isotherms for MIP/NIP@SiO 2 NPs.

Selectivity is a key parameter for any molecularly-imprinted polymers and directly affect the usability of the imprinted polymers for practical applications. The selectivity of the $\mathrm{MIP} @ \mathrm{SiO}_{2}$ NPs was tested with propranolol and pindolol as structural analogues of atenolol (Figure 5a). As seen in Figure $5 \mathrm{~b}$, the adsorption capacity of MIP@SiO${ }_{2}$ NPs for atenolol is much higher than the other molecules which strongly indicates the presence of selective cavities for atenolol on $\mathrm{MIP} @ \mathrm{SiO}_{2}$. Meanwhile, the adsorption capacities of NIP@SiO ${ }_{2}$ NPs are nearly same for all analogues implying the non-selective binding is predominant on the NIP@ $\mathrm{SiO}_{2}$ NPs.

(a)
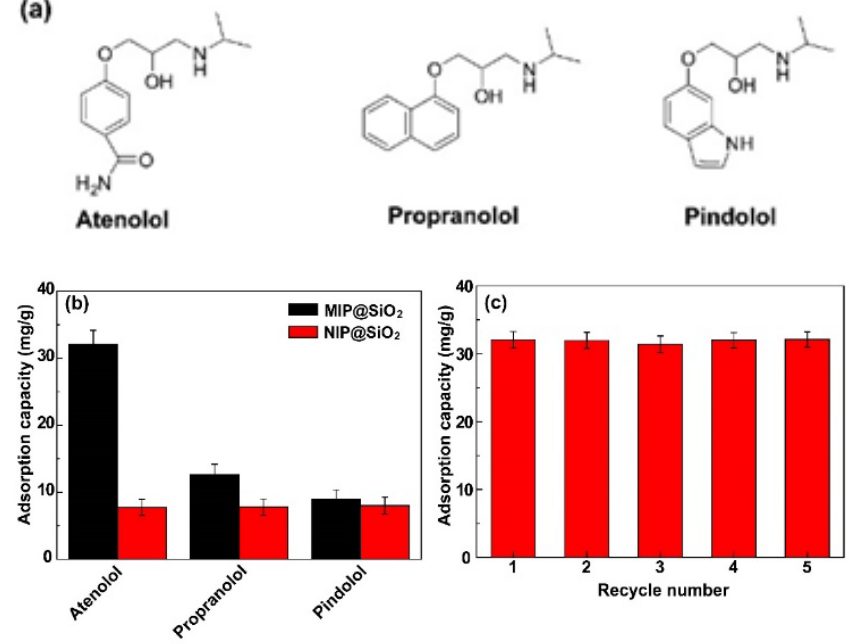

Figure 5. (a) Molecular structures of atenolol, propranolol and pindolol, (b) Selectivity of MIP/NIP@SiO 2 NPs and (c) Reusability of MIP@SiO NPs

The imprinting factor $\left(\alpha=Q_{M I P} / Q_{N I P}\right.$ where $Q_{M I P}$ and $Q_{N I P}$ are the adsorption capacity of the imprinted and non-imprinted particles, respectively [20]) and relative selectivity coefficient $\left(\beta=\alpha_{\text {atenolol }} / \alpha_{\text {analogue }}\right.$ where $\alpha_{\text {atenolol }}$ and $\alpha_{\text {analogue }}$ are the imprinting factor of atenolol and its analogues, respectively [21]) were also calculated to obtain more detail information of selectivity (Table 1). The results indicated that the imprinting factor for atenolol is larger than that of the others and the relative selectivity coefficient values are greater than 1 for MIP@SiO 2 NPs which is again the strongest indication that atenolol selectively binds due to the imprinting effect.

Table 1. The selectivity parameters of MIP/NIP@SiO 2 .

\begin{tabular}{lll}
\hline Molecule & $\boldsymbol{\alpha}$ & $\boldsymbol{\beta}$ \\
\hline Atenolol & 4.14 & - \\
\hline Propranolol & 1.63 & 2.54 \\
\hline Pindolol & 1.12 & 3.70 \\
\hline
\end{tabular}

Regeneration ability is another important parameter for any imprinted polymer. The reusability of the atenolol-imprinted particles was investigated via adsorption-desorption cycles and the results are given in Figure 5c. The adsorption capacity of $\mathrm{MIP} @ \mathrm{SiO}_{2}$ NPs is almost unchanged even after 5 adsorption-desorption cycles. As a result, the high selectivity and stability of the MIP@SiO 2 NPs indicate that it can be used in practical application such as separation and purification technologies.

3.4. Determination of atenolol in spiked artificial urine samples 
The artificial urine samples were spiked by atenolol with different atenolol concentration. Then atenolol was extracted with MIP@SiO ${ }_{2}$ and coupled with spectrofluorimetry. A good linearity was observed between 0.25-2.5 $\mu \mathrm{g} / \mathrm{mL}$ atenolol concentrations. Limit of detection (LOD) and limit of quantification (LOQ) were calculated to be $0.068 \mu \mathrm{g} / \mathrm{mL}$ and $0.228 \mu \mathrm{g} / \mathrm{mL}$, respectively. Moreover, the recoveries of atenolol varied between $98.6 \%$ and $100.1 \%$ for different spiked concentrations of atenolol with low standard deviations (less than 4.4\%) (Table 2) which indicated that the proposed method is accurate and practical.

Table 2. Recovery of atenolol from spiked artificial urine samples extract by MIP@SiO $\mathrm{NPs}_{\text {. }}(n=3)$.

\begin{tabular}{llll}
\hline $\begin{array}{l}\text { Added } \\
\text { concentration }(\boldsymbol{\mu g} / \mathbf{m L})\end{array}$ & $\begin{array}{l}\text { Found } \\
\text { concentration }(\boldsymbol{\mu g} / \mathbf{m L})\end{array}$ & $\begin{array}{c}\text { atenolol } \\
\text { Recovery, \% }\end{array}$ & $\begin{array}{l}\text { Relative standard } \\
\text { deviation, } \%\end{array}$ \\
\hline 0 & - & - & - \\
0.07 & $0.069 \pm 0.003$ & 98.6 & 4.34 \\
0.28 & $0.277 \pm 0.01$ & 98.9 & 3.61 \\
0.56 & $0.559 \pm 0.02$ & 99.8 & 3.57 \\
1.12 & $1.121 \pm 0.04$ & 100.1 & 3.56 \\
\hline
\end{tabular}

\section{Conclusion}

In summary, a novel atenolol-imprinted silica nanoparticles were synthesized via surface imprinting approach based on surface initiated free radical polymerization. The prepared imprinted nanoparticles showed high adsorption capacity, fast adsorption kinetic, high selectivity and stability. Moreover, the application of the imprinted nanoparticles was successfully carried out in selective extraction and determination of atenolol from artificial urine samples with high recovery and low standard deviation. The proposed method can be alternative candidate for determination of atenolol in biological samples.

\section{Acknowledgements}

The author would like to thank Prof. Dr. Zekiye SULUDERE for TEM analysis.

\section{References}

[1]. Odabaşı M., Uzun L., Baydemir G., Aksoy N.H., Acet Ö., Erdönmez D., "Cholesterol imprinted composite membranes for selective cholesterol recognition from intestinal mimicking solution", Colloids and Surfaces B: Biointerfaces, 163, (2018), 266-274.

[2]. Turan E., Şahin F., "Molecularly imprinted biocompatible magnetic nanoparticles for specific recognition of Ochratoxin A", Sensors and Actuators B: Chemical, 227, (2016), 668-676.

[3]. Ansell R.J., Kriz D., Mosbach K., "Molecularly imprinted polymers for bioanalysis: chromatography, binding assays and biomimetic sensors", Current Opinion in Biotechnology, 7, (1996), 89-94.
[4]. Dechtrirat D., Yarman A., Peng L., Lettau K., Wollenberger U., Mosbach K., Scheller F.W., 2 Catalytically Active MIP Architectures, in: S. Li, S. Cao, S.A. Piletsky, A.P.F. Turner (Eds.) Molecularly Imprinted Catalysts, Elsevier, Amsterdam, 2016, pp. 19-34.

[5]. Mo G., Qin D., Jiang X., Zheng X., Mo W., Deng B., "A sensitive electrochemiluminescence biosensor based on metal-organic framework and imprinted polymer for squamous cell carcinoma antigen detection", Sensors and Actuators B: Chemical, 310, (2020), 127852.

[6]. Regal P., Díaz-Bao M., Barreiro R., Cepeda A., Fente C., "Application of molecularly imprinted polymers in food analysis: clean-up and chromatographic improvements", Open Chemistry, 10, (2012), 766-784.

[7]. Cormack P.A.G., Elorza A.Z., "Molecularly imprinted polymers: synthesis and characterisation", Journal of Chromatography B, 804, (2004), 173-182.

[8]. Gupta P., Goyal R.N., "Amino Functionalized Graphene Oxide and Polymer Nanocomposite Based Electrochemical Platform for Sensitive Assay of AntiDoping Drug Atenolol in Biological Fluids", Journal of The Electrochemical Society, 163, (2016), B601-B608.

[9]. Hasanah A.N., Dwi Utari T.N., Pratiwi R., "Synthesis of Atenolol-Imprinted Polymers with Methyl Methacrylate as Functional Monomer in Propanol Using Bulk and Precipitation Polymerization Method", Journal of Analytical Methods in Chemistry, 2019, (2019), 9853620.

[10]. Kannappan V., Mannemala S.S., "Simultaneous enantioseparation and purity determination of chiral 
switches of amlodipine and atenolol by liquid chromatography", Journal of Pharmaceutical and Biomedical Analysis, 120, (2016), 221-227.

[11]. Shah J.V., Patel D.P., Shah P.A., Sanyal M., Shrivastav P.S., "Simultaneous quantification of atenolol and chlorthalidone in human plasma by ultra-performance liquid chromatography-tandem mass spectrometry", Biomedical Chromatography, 30, (2016), 208-216.

[12]. Yilmaz B., Arslan S., "Determination of Atenolol in Human Urine by Gas Chromatography-Mass Spectrometry Method", Journal of Chromatographic Science, 49, (2011), 365-369.

[13]. Arvand M., Vejdani M., Moghimi M., "Construction and performance characterization of an ion selective electrode for potentiometric determination of atenolol in pharmaceutical preparations", Desalination, 225, (2008), 176-184.

[14]. Damiani P.C., "Determination of atenolol in human urine by emission-excitation fluorescence matrices and unfolded partial least-squares with residual bilinearization", Talanta, 85, (2011), 1526-1534.

[15]. Sapir A., Shalev A.H., Skalka N., Bronshtein A., Altstein M., "Development of an enzyme-linked immunosorbent assay and a beta-1 adrenergic receptorbased assay for monitoring the drug atenolol", Environmental Toxicology and Chemistry, 32, (2013), 585-593.

[16]. Mellon V., Rinaldi D., Bourgeat-Lami E., D'Agosto F., "Block Copolymers of $\gamma$ -
Methacryloxypropyltrimethoxysilane and Methyl Methacrylate by RAFT Polymerization. A New Class of Polymeric Precursors for the Sol-Gel Process", Macromolecules, 38, (2005), 1591-1598.

[17]. Lopez C., Claude B., Morin P., Pelissou M., Pena R., Max J.-P., Ribet J.-P., "Synthesis and study of a molecularly imprinted polymer for specific solid-phase extraction of vinflunine and its metabolite from biological fluids", Journal of Separation Science, 34, (2011), 1902-1909.

[18]. Zengin A., Tamer U., Caykara T., "A SERS-Based Sandwich Assay for Ultrasensitive and Selective Detection of Alzheimer's Tau Protein", Biomacromolecules, 14, (2013), 3001-3009.

[19]. Bilici M., "Synthesis of a Novel Molecularly Imprinted Polymer for the Sensitive and Selective Determination of Artemisinin in Urine Samples Based on Solid-Phase Extraction (SPE) and Determination with HighPerformance Liquid Chromatography (HPLC)", Analytical Letters, (2020), 1-17.

[20]. Gorbani Y., Yılmaz H., Basan H., "Spectrofluorimetric determination of atenolol from human urine using highaffinity molecularly imprinted solid-phase extraction sorbent", Luminescence, 32, (2017), 1391-1397.

[21]. Zengin A., Badak M.U., Aktas N., "Selective separation and determination of quercetin from red wine by molecularly imprinted nanoparticles coupled with HPLC and ultraviolet detection", Journal of Separation Science, 41, (2018), 3459-3466.. 